\title{
AN APPROACH TO AN INTELLIGENT SCANNING OF THE MACHINE TOOL WORKSPACE
}

\begin{abstract}
Modern 3D scanners can measure the geometry with high accuracy and within a short time. In turn, currently produced CNC machine tools allow for very accurate manufacturing; however, processes beyond the machining cycle remain time-consuming. This paper presents the idea and experimental tests of the scanning system in the $\mathrm{CNC}$ machine, which allows to speed up on-machine measurements, align clouds of 3D data points with an accuracy close to that of the machine itself, and finally set the workpiece coordinate system for machining. This modern approach is in line with Industry 4.0, combining the terms of data processing, machine vision, manufacturing automation, and human-machine interfaces. The future implementation of the proposed system as an interchangeable tool will allow performing autonomous measurements, inspection, and supervision of the workspace, without engaging the machine operator. The system calibration and experimental results using the industrial 3D scanner and $\mathrm{CNC}$ machine are described.
\end{abstract}

\section{INTRODUCTION}

\subsection{STATE OF THE ART}

Non-contact measurement methods, such as 3D laser scanners or structured light scanners, are increasingly used in the industry for inspection tasks and in reverse engineering [1]. These measurements are becoming faster and allow continuous monitoring of manufacturing processes, among others in the automotive industry [2-4]. Optical measurements are also used in rapid prototyping, archaeology, education, medicine, or design [5-9].

The scanner can be used with a studio stand or a desk stand for manual operations, or it can be mounted on an industrial robotic arm in automated applications. By changing the position of the scanner head in space, it is possible to measure objects of different sizes and shapes from different directions. Matching scans is usually done with the use of reference

\footnotetext{
${ }^{1}$ Faculty of Mechanical Engineering and Mechatronics, West Pomeranian University of Technology in Szczecin, Poland

*Email: lukasz.marchewka@zut.edu.pl https://doi.org/10.36897/jme/136414
} 
points (so-called markers) placed on the scanned object, which allows making the measurement independent of the industrial robot low accuracy and even more of its coordinate system [10].

Structured light scanners are characterized by a high resolution and high accuracy of a few to several micrometers [11-13]. Even higher accuracy is difficult to obtain due to varying environmental factors (mainly ambient temperature), or the manufacturing accuracy of the calibration pattern $[14,15]$. Currently, scanners are only regulated by the VDI/VDE 2634 standard relating to three-dimensional optical measuring systems, whose main function is based on triangulation [16]. The standard concerns measurements of the defined geometric patterns measured in different orientations and positions [17].

Laser scanners are also used for non-contact geometry measurements, giving similar results in terms of accuracy to the scanners based on structured light projection [18-21]. The main difference is the much shorter time for simultaneous scanning of a large area in the case of structured light scanners, while laser scanning is done additively by moving a point, a stripe, or a mesh over the scanned surface.

The authors of the paper [22] used the optical 3D scanner to assess the accuracy of the machine tool body casting with respect to the reference CAD model. This method allowed determining whether the size of the allowances for all surfaces is sufficient for appropriate machining and thus minimizing material waste by $4.5-9.6 \%$.

In other papers $[23,24]$, the authors present a system for basing a workpiece, located outside the machine tool but related to its machine coordinate system. Determination of such a relation is performed using necessary additional reference points (markers) and this solution is presented as an alternative to the expensive and delicate touch probe. The disadvantage of such a system is that these points must be fixed permanently on the machine table in known positions relative to the machine coordinate system because the accuracy of basing procedures depends on them. In the paper, the authors also mention the idea of mounting such a scanner inside the machine tool as an integral component. There are also metrology systems, including industrial measurement scanners, mounted in the tool holder of the machine tool, but not linked with the machine coordinate system or the control system [25-27]. These systems are used for inspection of the workpiece or reverse engineering tasks and their capabilities are limited to measuring the geometry of the workpiece in its own local reference system. The authors of the paper [28] proposed the use of a scanner as a tool for basing the workpiece using an edge detection algorithm. The scanner control system transmits information about edge detection to the machine control system. However, this paper does not reveal any crucial information about the calibration and integration of both independent systems and other important functionalities that we propose in our paper.

Positioning of the workpiece in relation to the machine coordinate system of the CNC lathe was proposed in [29]. The authors used fiducial markers with well-known geometry and location in the machine in order to find the relation of every single scan. This approach has the same fundamentals as traditional scanning techniques with flat coded markers, used to find the spatial transformation of each scan. Moreover, in this method, at least one fiducial feature must be visible while scanning the workpiece which limits scanning possibilities. The authors also considered the registration of additional machine movements to define the transformation of the scan related to fiducial features. 
In the solution proposed in [30], the 3D scanner was mounted in the tool holder of the cartesian CNC machine in order to simplify and speed up the geometry measurements. By executing planned machine movements, the workpiece mounted on the machine table could be fully scanned. However, the coordinate systems of both the 3D scanner and CNC machine tool were not related, therefore, measured points needed to be transformed and expressed within the scanner workspace. Due to the kinematic structure of the machine allowing only translation movements, the scan alignment was a simple task.

There are known patented solutions known that have apparently similar functionality to ours, but they differ fundamentally in terms of flexibility, scanning techniques, and do not explain any details about the calibration of optical devices related to machine coordinate systems. In the solution [31], scanning is performed using a single laser beam moving along only one controlled axis, which greatly simplifies geometry reconstruction. According to the idea, the device indicates the mass distribution and supports the initial centering of crankshaft axes, which is finally performed manually before machining using the screw adjustments.

Another invention described in [32] involves simultaneous camera observation and visualization of a planned cutting process of metal sheets. The solution uses a camera image on which a model of the machining process is overlaid and allows the operator to assess the correctness of the planned cutting trajectories. As the workpiece moves within the camera field of view, the images require a group of reference points fixed around the workpiece and permanently visible, which is opposite to our markerless solution. Furthermore, the calibration is simplified and only the position of the camera relative to the workpiece is derived. The kinematic structure of the machine tool and the relationship between its axes and the camera cannot be determined in this way.

\subsection{AIMS AND MOTIVATION}

Industrial use of the above-mentioned methods as well as contact measurements using the CMM results in a longer manufacturing process by increasing the duration time of processes beyond the machining cycle (handling, temporary storage, inspection). The workpiece to be measured must be disassembled from the machine, cleaned, and transported to the measuring station. In addition, markers are problematic to use in the CNC machine space due to the numerous contaminants.

In most cases, on-machine measurements are currently carried out by manually controlled touch probes. The machine is not able to locate features within its workspace automatically, and in the case of a new part fitted before machining, there is a risk to damage the probe by an inexperienced operator. The measurements are relatively long for only a few points, making it impossible to carry out a comprehensive inspection of the major part of the workpiece. Finally, safety issues need to be considered as the operator usually has to enter the machine's workspace in order to assess the probe positions.

The aim of this paper is the practical verification of the concept, which assumes the use of fast 3D vision scanning and machine motion accuracy to automatically match the scans of any object moving and rotating in the workspace without using reference points. Moreover, by assuming known machine structure and motions, all the transformed point clouds should be expressed in machine coordinate systems, allowing further non-contact inspections. 


\subsection{PAPER CONTRIBUTION}

The proposed innovation indicates new directions in the use of metrology systems in machine tools. The use of markers is an inflexible and impermanent solution because the places of applying the markers have to be selected individually for each measured element and require a properly prepared surface. In contrast to related work, our innovative approach concerns integrating the 3D scanning system with the kinematic structure of the machine tool, which uses only the readout of the machine positions sensors to align the point clouds. The implementation of calibration procedures will create a common, three-coordinate reference system for both devices, allowing fast, intuitive, and safe measurements of any point in the machine coordinate system, and scanning from any direction available on the machine without the use of markers. In addition, implementation of an artificial intelligence algorithm will allow rapid classification of objects and prediction of the possibility of collision between the scanning tool and machine components and elements placed on the machine table, as well as planning of measurement strategy and trajectory. It could also be used to pre-analyse, detect and reject difficult-to-scan surfaces (which scanners do not do in practice).

\section{MATERIALS AND METHODS}

\subsection{CONCEPT OF THE PROPOSED SYSTEM}

The presented solution is partially the subject of our patent application. According to our idea, the independent coordinate system of the 3D scanner workspace can relate to the kinematic chain of the CNC machine tool by appropriate calibration procedures. Then, any further movements of the machine bodies read from the position sensors (or directly from $\mathrm{G}$-code) transform the 3D scanner workspace. One of the body configurations must be used also to define in the $\mathrm{CNC}$ program the local machine coordinate system (called further LMCS). For a predefined kinematic structure of the machine tool (but with unknown parameters), the scanning software creates an accurate parameterized model of this structure based on a set of properly prepared basic movements of machine bodies. During normal system operation, each partial scan is back-transformed according to the modelled kinematic chain and positioned in the LMCS. As a result, all partial scans should compose into a single point cloud, representing e.g. the workpiece. Theoretically, such a calibration can be applied to any machine tool with any number of degrees of freedom.

The accuracy of individual scans alignment depends directly on the accuracy of the machine tool itself, the accuracy of the scanner, and the durability and stability of its mounting on the machine tool. However, we estimated the errors of the proposed system by analysing the accuracy of the point clouds assembly. According to the VDI/VDE 2634 standard for optical triangulation measurements [16], only the results of the whole measuring system are taken into account, instead of considering each individual scanner component or image data analysis methods. 


\subsection{SYSTEM PREPARATION AND CALIBRATION}

As we mentioned, in the classical approach an accurate and unambiguous scans alignment is performed using a set of markers, which is however impractical and timeconsuming on the $\mathrm{CNC}$ machine tools. Alternatively, we decided to use planned basic movements of the machine tool, including rotations and translations of each available axis in the field of view of the 3D scanner. As shown in Fig. 1, we chose the 5-axis DMG DMU 60 MonoBlock machine tool (DMG Mori Seiki Co., Ltd., Tokyo, Japan) and a GOM Atos Triple Scan optical scanner (GOM GmbH, Braunschweig, Germany) with an accuracy of $10 \mu \mathrm{m}$ for a diagonal field of view of $560 \mathrm{~mm}$ [33].

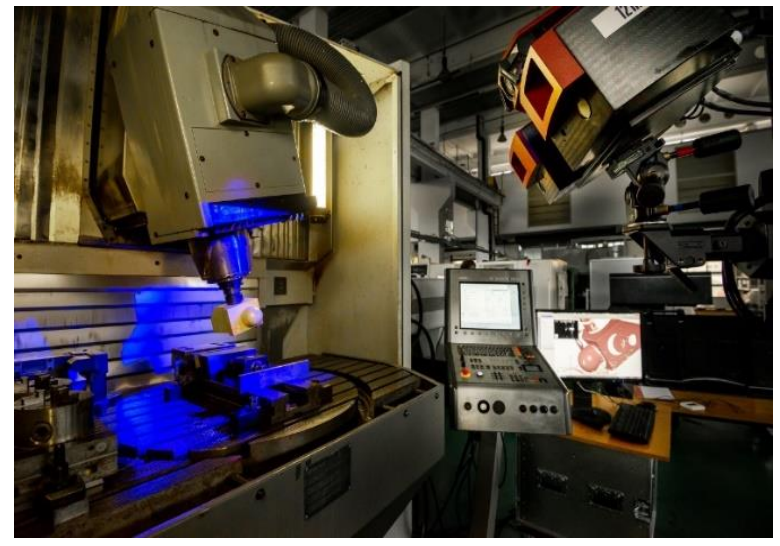

Fig. 1. The test stand consisting of DMG DMU MonoBlock and industrial 3D scanner GOM Atos Triple Scan $12 \mathrm{M}$

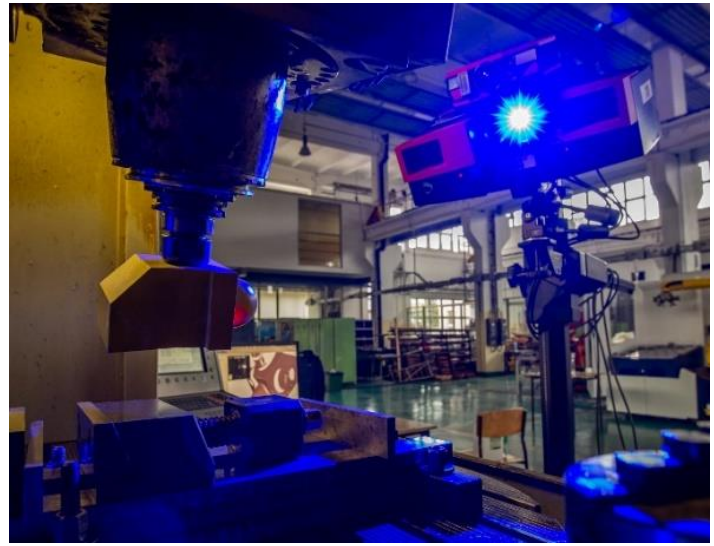

Fig. 2. The sample object in a cubic form containing basic elements such as spheres, cylinders, surfaces, mounted on the standard SK40 tool holder

We prepared a steel sample object (shown in Fig. 2) so that it contains primitive geometric shapes i.e. flat surfaces, cylinders, and convex spheres. The sample object was painted with a homogeneous matt paint and covered with a thin matting spray containing $\mathrm{TiO}_{2}$ to avoid potential scanning errors. We assumed that the object should be able to transform in as much as possible degrees of freedom. As the table on the DMU milling machine only allows vertical movement and rotation around its $\mathrm{Z}$ axis, we decided that it would be more non-trivial and interesting to attach the object to the spindle using an SK40 tool holder. The construction of the column allowed the component to move in X, Y directions, and rotations in the spindle axis and the tilting axis $B$. Unfortunately, while preparing the experiment, we had to reject spindle rotations due to the low accuracy of the angular positioning (about $\pm 0.5^{\circ}$ ) and leave it electrically locked in one position.

According to Fig. 3, the scanner was fixed permanently in an unknown position relative to the machine tool, but so that it could observe movements of the sample object (with representing point $p$ ). The proposed method assumes a general knowledge of the kinematic structure, but no exact parameters values of the kinematic structure. Since the 3D scanner and the machine tool are independent, it was necessary to precede a properly planned calibration procedure, merging the $S$ and $M$ coordinate systems of both devices. In the CNC program, we 
determined our own local $L M C S$ coordinate system, to which the initiating $X, Y, B$ values corresponded $\left(\mathrm{X}: 0 \mathrm{~mm}, \mathrm{Y}: 0 \mathrm{~mm}, \mathrm{~B}:-16^{\circ}\right)$. We attached a single circular marker $m$ near the scanned sample object in an unknown position. Then, the machine tool performed a series of translations along the $X$ and $Y$ axes individually in the range of $\pm 200 \mathrm{~mm}$. Then, for the value $X=0$ the machine performed a series of rotations around the $B$ axis in the range of $\pm 30^{\circ}$, while gradually moving along the $Y$ axis. A scheme of marker displacements is shown in Fig. 4. Positions of the marker $m$ were captured in the scanner coordinate system and exported to Matlab (The MathWorks, Inc., Natick, USA) in one text file. The marker $m$, used only in the calibration process, was removed before further experiments.

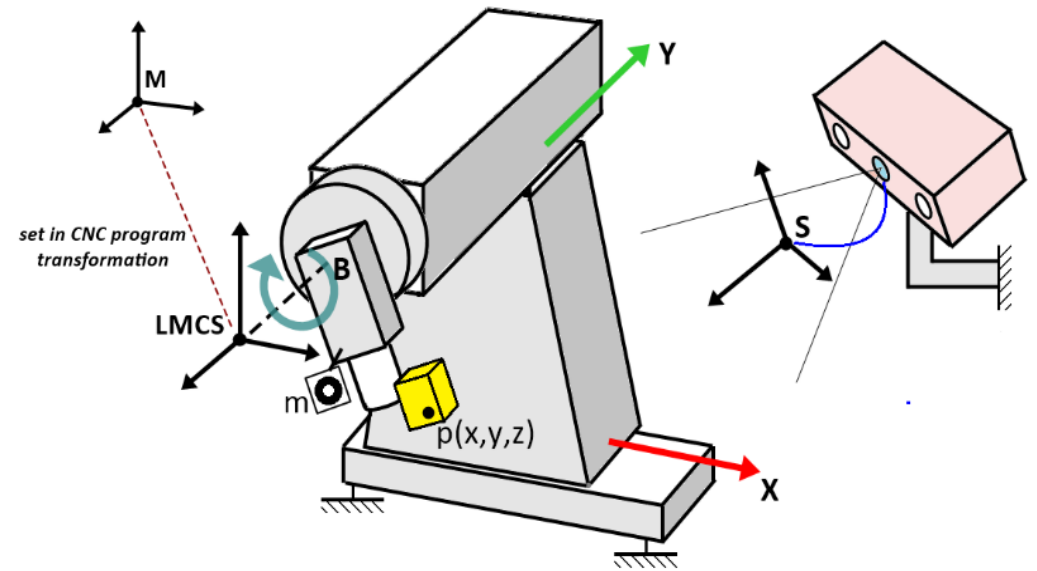

Fig. 3. General scheme of the test stand and relating reference systems. The calibration marker $m$ and the sample object are attached to the moveable spindle column. The $S$ coordinate system is independent of the local machine coordinate system (LMCS) as well as of the machine coordinate system $(M)$

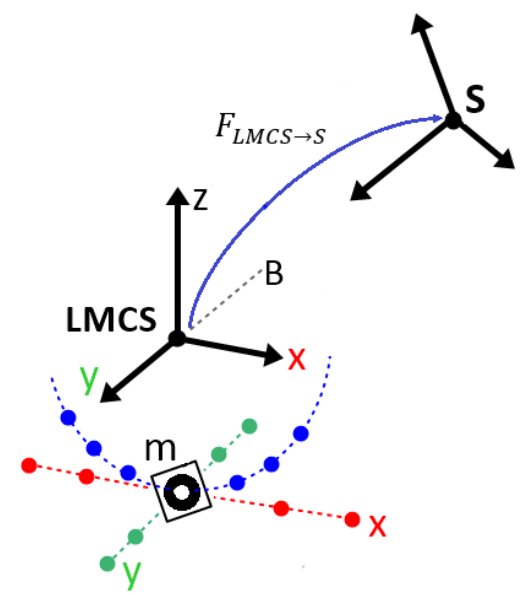

Fig. 4. Simplified scheme of calibration movements for each axis independently.

Each point indicates the position of marker $m$ captured by the 3D scanner in its own coordinate system $(S)$

In Matlab, we fitted a cylinder and two straight lines to the captured points, which allowed us to construct the geometric equivalent of the LMCS but still expressed in the $S$ coordinate system. We also verified the correctness of our calculations in the GOM Atos software by reconstructing the $L M C S$ (Fig. 4). Then, we determined the simple and inverse transformation between the $S$ and reconstructed $L M C S$, using rigid body transformation and SVD algorithm [34], which finally allowed us to transform the scanner space to the machine tool LMCS coordinate system.

\subsection{EXPERIMENTAL MEASUREMENTS OF SCANS ALIGNMENT}

After calibration, without any marker attached, we made a series of combined $X, Y$ translations, and $B$ rotations of the sample object while scanning its surfaces. As we mentioned, the partial scans obtained for each specified position of the machine tool did not form a consistent geometry in $S$, as shown in Fig. 5 and Fig. 6, which is obvious for moving objects from the point of view of the fixed scanner. 


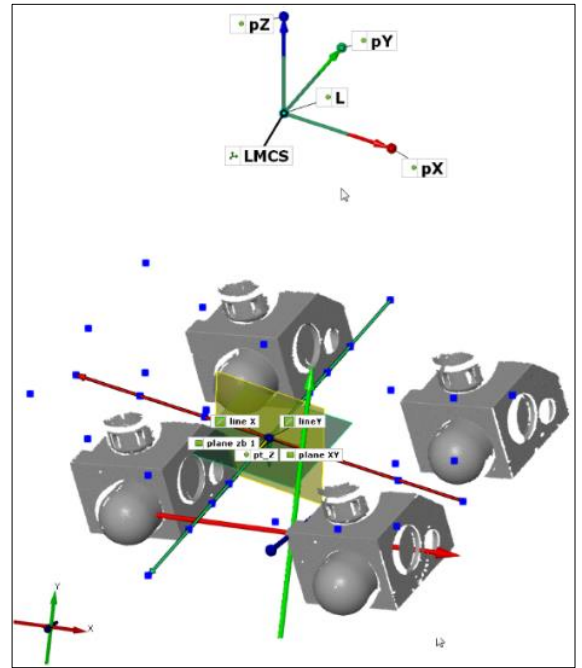

Fig. 5. Four mismatched scans representing the sample object, visible in the scanner coordinate system. Colored dots indicate captured positions of calibration marker m, which allowed creating local X, Y axes directions and origin of LMCS

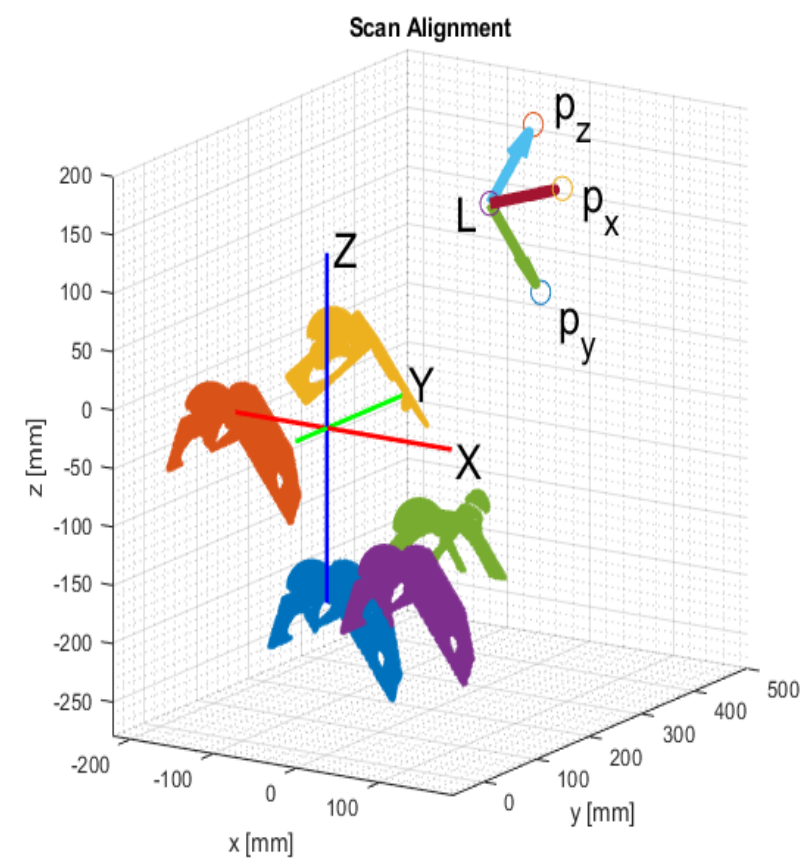

Fig. 7. Mismatched points clouds and reconstructed points $p x, p y, p z, p L$, indicating local machine coordinate system, imported to Matlab

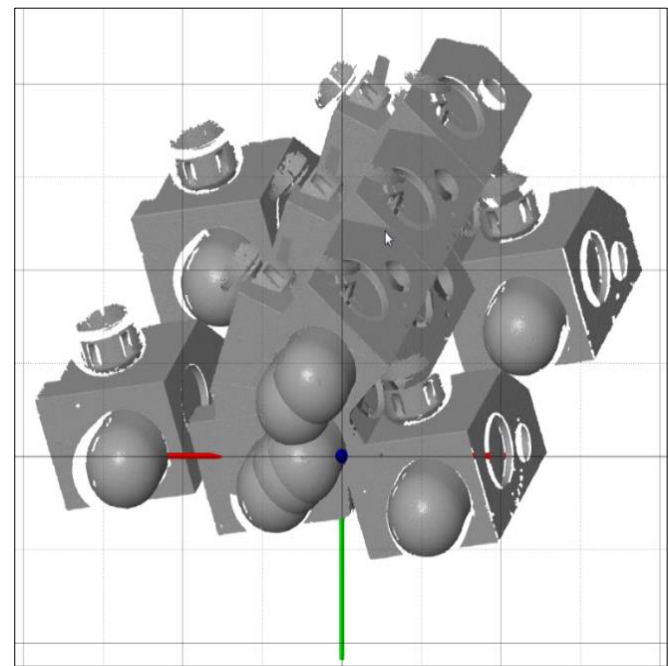

Fig. 6. Collection of mismatched scans representing the sample object moving in the scanner coordinate system

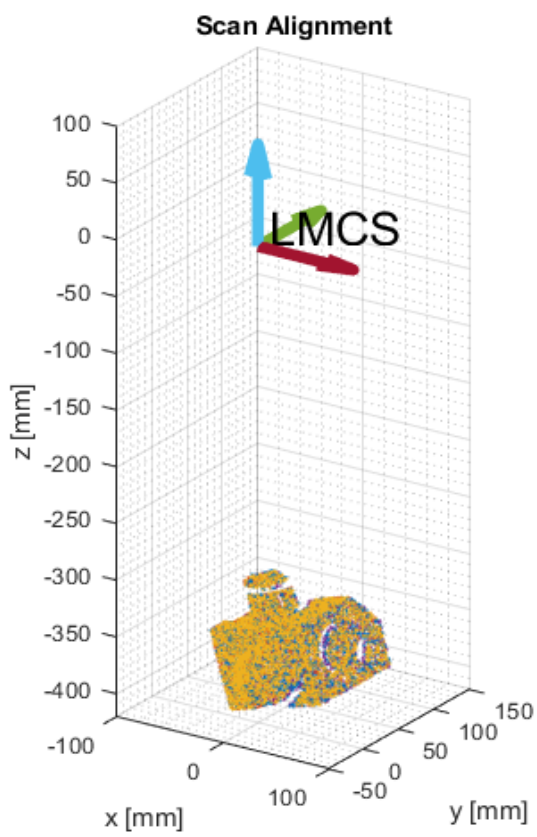

Fig. 8. Merged scans after applying scanner-tomachine transformation and reconstruction of machine bodies movements for each scan

Our task was to prove, that by applying inverse transformations to those planned in the CNC program and using the calibrated kinematic structure of the machine, it was possible to combine all the partial scans with high accuracy. All the 3D point clouds, the machine tool positions, and angles were imported into Matlab (Fig. 7), which allowed us to calculate and to apply the inverse transformation of each scan to the $L M C S$, using the general equation: 


$$
\left[\boldsymbol{P}^{\prime}\right]_{4 x n}=\left[\boldsymbol{R}_{\boldsymbol{y}}\right]_{4 x 4}\left[\boldsymbol{t}_{x y}\right]_{4 x 4}\left[F_{S \rightarrow L M C S}\right]_{4 x 4}[\boldsymbol{P}]_{4 x n}
$$

where:

$$
F_{S \rightarrow L M C S}=\left[\begin{array}{cccc}
\boldsymbol{R}^{-1} & \boldsymbol{R}^{-1}(-\boldsymbol{t}) \\
0 & 0 & 0 & 1
\end{array}\right]
$$

is the homogeneous transformation between the scanner and local machine coordinate systems, including its rotation and translation:

$$
\boldsymbol{R}=\left[\begin{array}{lll}
v_{x_{x}} & v_{x_{y}} & v_{x_{z}} \\
v_{y_{x}} & v_{y_{y}} & v_{y_{z}} \\
v_{z_{x}} & v_{z_{y}} & v_{z_{z}}
\end{array}\right], \quad \boldsymbol{t}=\left[\begin{array}{c}
L_{x} \\
L_{y} \\
L_{z}
\end{array}\right],
$$

obtained from three vectors constructed based on points $p x, p y, p z$ and $p L$ using equations:

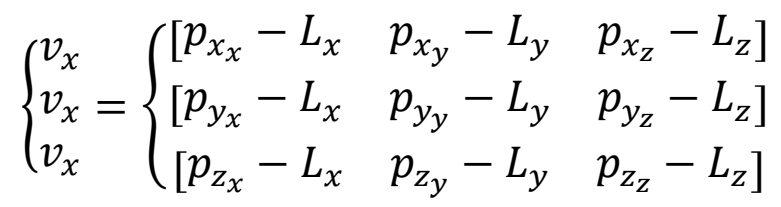

and normalized to three versors as follows:

$$
\left\{\begin{array}{l}
v_{x} \\
v_{x} \\
v_{x}
\end{array}=\left\{\begin{array}{l}
\frac{v_{x}}{\sqrt{\sum_{k=1}^{3} v_{x_{k}}^{2}}} \\
\frac{v_{y}}{\sqrt{\sum_{k=1}^{3} v_{y_{k}}^{2}}} \\
\frac{v_{z}}{\sqrt{\sum_{k=1}^{3} v_{z_{k}}^{2}}}
\end{array}\right.\right.
$$

The last two transformations of points $P$ in the equation (1) describe their rotations around the $\mathrm{B}$ axis and translations in $\mathrm{X}$ and $\mathrm{Y}$ axes in LMCS

$$
\boldsymbol{t}_{x y}=\left[\begin{array}{llll}
1 & 0 & 0 & t_{x} \\
0 & 1 & 0 & t_{y} \\
0 & 0 & 1 & 0 \\
0 & 0 & 0 & 1
\end{array}\right], \boldsymbol{R}_{\boldsymbol{y}}=\left[\begin{array}{cccc}
\cos \alpha & 0 & \sin \alpha & 0 \\
0 & 1 & 0 & 0 \\
-\sin \alpha & 0 & \cos \alpha & 0 \\
0 & 0 & 0 & 1
\end{array}\right],
$$

written in the correct order, dependent on the kinematic structure of the machine tool. We presented the results of selected transformations in Fig. 8 and a closer view in Fig. 9.

Unfortunately, the intentionally disabled spindle axis did not allow us to scan the opposite surfaces of the sample object, therefore we could not measure the most obvious linear dimensions. Instead, we proposed an error analysis for the relative center positions of the spheres, as well as a flatness and parallelism analysis of selected planes, which together allowed us to evaluate overall system abilities to merge the point clouds. 


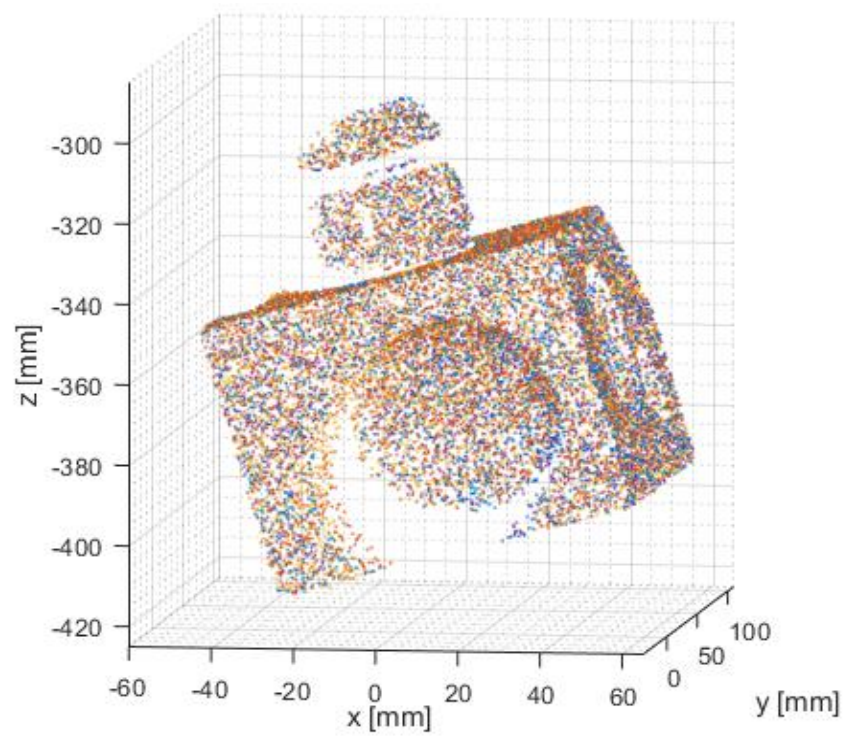

Fig. 9. Close view of the scans after applying transformations based on machine movements. For a better visibility, every tenth point is displayed

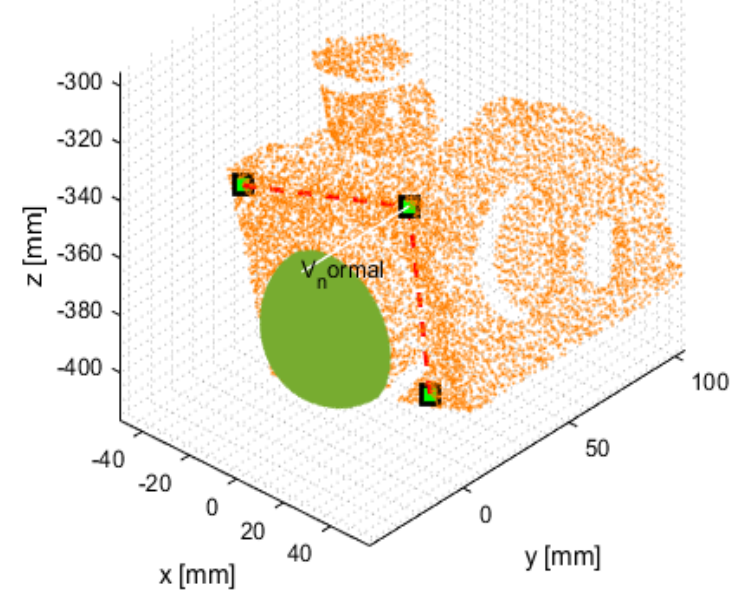

Fig. 10. Example of data selection before sphere model alignment using cutting off by plane method

In Matlab, we isolated data points of all scans by modelling the cut-off planes, and then we fitted models of spheres and planes to them using the least squares method. Due to the socalled edge effect, which typically occurs for edges of occluded objects and causes incorrect surface reconstruction, we applied additional data filtering of each isolated surface for the most outlying points over the value of the standard deviation. Then, we repeated planes alignment to the remaining 3D data points for each individual scan.

\subsection{RESULTS AND DISCUSSION}

After applying the proposed spatial transformations resulting in one composed 3D model of the sample object, we carried out an error analysis of all the combined surfaces and we obtained the following results. In Figs. 11-14, for better visibility, the calculated center positions of all spheres were accumulated inside another sphere, which center point relates to the mean center value, and its diameter is described by the outermost center point.

During data analysis, we rejected the scan no. 11 due to the appearance of a coarse error of $0.1 \mathrm{~mm}$ in comparison with the other scans, which may have been caused by a sudden change in measurement conditions or an unknown software issue. The position error of the spheres centres for the $\mathrm{x}$-axis and $\mathrm{z}$-axis is about $\pm 0.01 \mathrm{~mm}$, while for the $Y$-axis it is about $\pm 0.035 \mathrm{~mm}$. The results seen in Fig. 11 and Fig. 12 are comparable with the calculated standard deviations for each axis. Table 1 also shows the radius of the fitted spheres for the individual scans and its average value of $0.005 \mathrm{~mm}$ (while the reference radius measured on CMM is $25.002 \mathrm{~mm}$ ), which only confirmed the accuracy of the scanner itself. Similar results were obtained for the scans combined using only linear motion (no rotations), as shown in Fig. 13 and Fig. 14. 
Table 1. Reconstructed positions and radius of the spheres fitted to the selected $3 \mathrm{D}$ data point

\begin{tabular}{|c|c|c|c|c|c|}
\hline & & \multicolumn{3}{|c|}{$\begin{array}{c}\text { Fitted sphere } \\
\text { Center positions in LMCS }\end{array}$} & \multirow{2}{*}{$\begin{array}{c}\begin{array}{c}\text { Fitted } \\
\text { sphere } \\
\text { Radius }\end{array} \\
\mathrm{R}[\mathrm{mm}]\end{array}$} \\
\hline & $\begin{array}{c}\text { Scan } \\
\text { no. }\end{array}$ & $\mathrm{x}[\mathrm{mm}]$ & $\mathrm{y}[\mathrm{mm}]$ & $\mathrm{z}[\mathrm{mm}]$ & \\
\hline \multirow{4}{*}{ 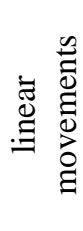 } & 1 & 13.294 & -8.948 & -372.794 & 25.001 \\
\hline & 2 & 13.292 & -8.946 & -372.801 & 25.007 \\
\hline & 3 & 13.285 & -8.973 & -372.804 & 25.003 \\
\hline & 4 & 13.294 & -8.977 & -372.800 & 25.000 \\
\hline \multirow{15}{*}{ 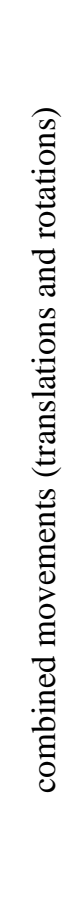 } & 5 & 13.296 & -8.993 & -372.801 & 25.003 \\
\hline & 6 & 13.302 & -8.986 & -372.789 & 25.006 \\
\hline & 7 & 13.300 & -8.960 & -372.803 & 25.006 \\
\hline & 8 & 13.292 & -8.951 & -372.797 & 25.004 \\
\hline & 9 & 13.290 & -8.966 & -372.790 & 25.004 \\
\hline & 10 & 13.290 & -8.997 & -372.795 & 24.999 \\
\hline & 11 & - & - & - & - \\
\hline & 12 & 13.289 & -8.954 & -372.791 & 25.005 \\
\hline & 13 & 13.289 & -8.939 & -372.799 & 25.004 \\
\hline & 14 & 13.292 & -8.946 & -372.799 & 25.005 \\
\hline & 15 & 13.290 & -8.958 & -372.798 & 25.007 \\
\hline & 16 & 13.300 & -9.005 & -372.812 & 25.006 \\
\hline & 17 & 13.294 & -8.960 & -372.795 & 25.005 \\
\hline & 18 & 13.298 & -8.936 & -372.795 & 25.008 \\
\hline & 19 & 13.302 & -8.945 & -372.791 & 25.008 \\
\hline \multicolumn{2}{|c|}{ Mean value } & 13.294 & -8.963 & -372.797 & 25.005 \\
\hline \multicolumn{2}{|c|}{ Std. } & 0.005 & 0.020 & 0.006 & 0.003 \\
\hline
\end{tabular}

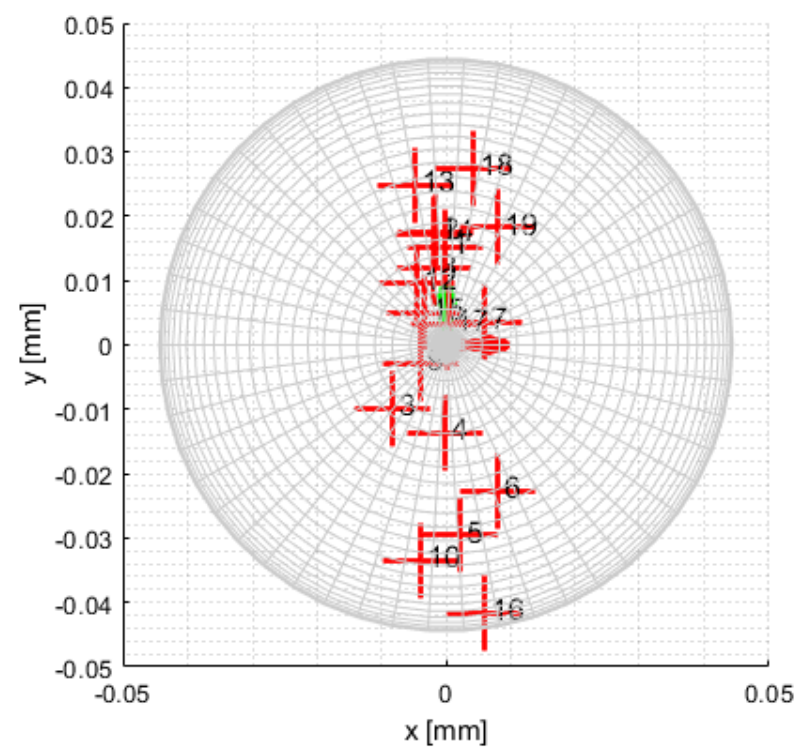

Fig. 11. Position errors of spheres centres visible on the $X Y$ plane for combined movements

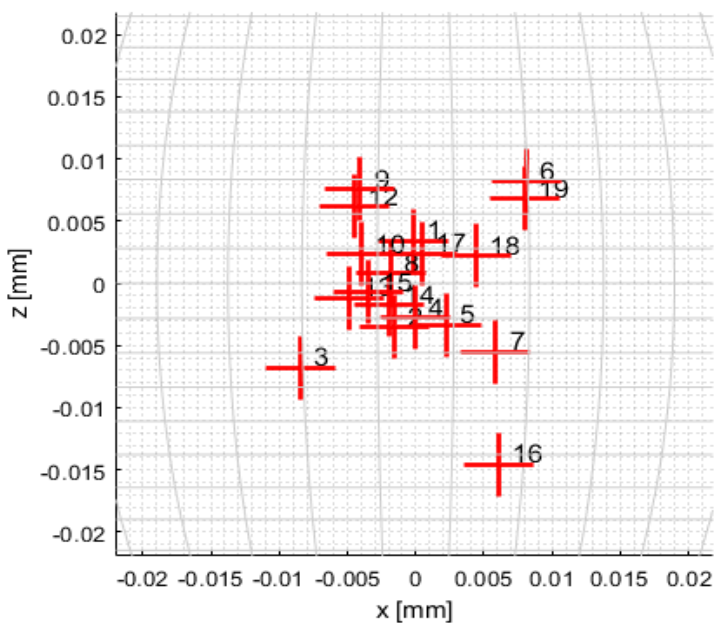

Fig. 12. Position errors of spheres centres visible on the $X Z$ plane for combined movements

We observed an error in the position of the centre of the fitted sphere in the $Y$-axis more than 3 times larger than for the other axes. In our research, we assumed that the positioning accuracy of the machine tool is at least $0.005 \mathrm{~mm}$ and took its movements as reference values to merge the scans. However, the error distribution is noticeably consistent with the direction of the $Y$-axis in the reconstructed LMCS coordinate system of the machine, which may be due to fatal positioning errors in this axis. We rejected the possibility that the 3D scanner generated errors due to the significantly different orientation of the scanning head relative to the machine coordinate system (the difference in angles in the $X, Z$ axes was more than $25^{\circ}$ ). Future research must be preceded by machine tool accuracy measurements using laser interferometers that can confirm our current assumptions. Moreover, to identify the source of the error, measurements would have to be repeated on the machine with compensation 
implemented for systematic and easily modeled errors. We are also not fully convinced that we have completely eliminated spindle rotation. The spindle had no mechanical locking capability, and the holding torque of the BLDC drive alone may not have been sufficient to hold the scanned part fixed, especially during acceleration and deceleration of the machine axis. In addition, the scanner was mounted on an aluminum tripod, completely independent of the CNC machine tool bodies, with a considerable extension and height. We cannot exclude small displacements of the scanning head relative to the column and spindle of the CNC machine tool, taking into account the long duration of the measurements.

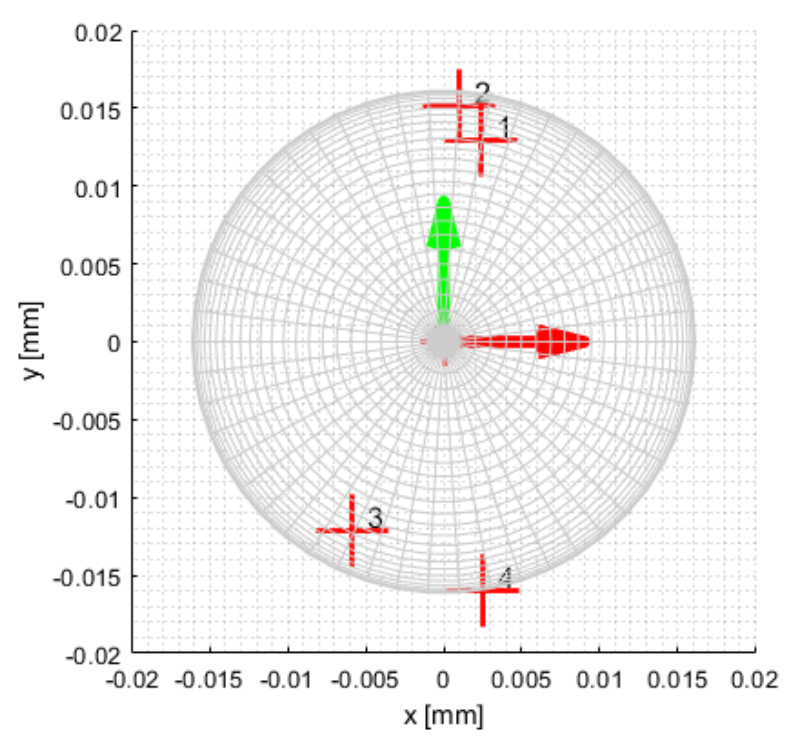

Fig. 13. Position errors of spheres centres visible on the $X Y$ plane for linear movements

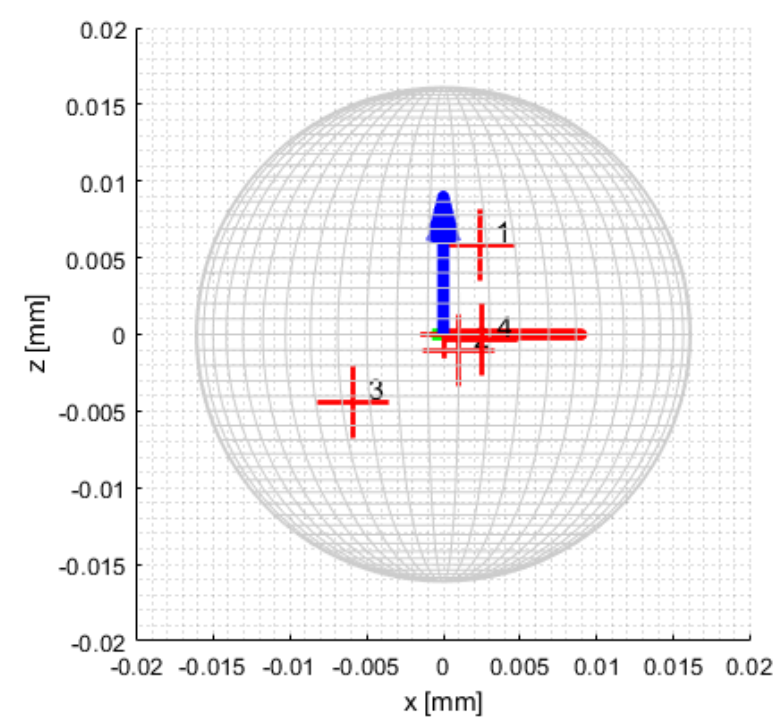

Fig. 14. Position errors of spheres centres visible on the $X Z$ plane for linear movements 
For all combined data points, representing the same flat surface of the sample object (Fig. 15 and Fig. 16), we fitted one reference plane and calculated one flatness indicator according to the GD\&T system [35]. Then, we used the normal vector of the reference plane to calculate the parallelism of each individual plane with respect to the reference plane. The obtained results are shown in Table 2. The parallelism indicators did not exceed $0.03 \mathrm{~mm}$, and its average value is about $0.015 \mathrm{~mm}$, which is close to the value of the standard deviation.

Table 2. Parallelism and flatness indices of the selected

flat surface combined of all transformed scans

\begin{tabular}{|c|c|c|c|c|}
\hline & $\begin{array}{c}\text { Scan } \\
\text { no. }\end{array}$ & $\begin{array}{l}\text { Parallelism } \\
{[\text { [mm] }} \\
\text { individual }\end{array}$ & $\begin{array}{l}\text { Flatness } \\
{[\mathrm{mm}]} \\
\text { combined }\end{array}$ & $\begin{array}{c}\text { Std. } \\
{[\mathrm{mm}]} \\
\text { combined }\end{array}$ \\
\hline \multirow{4}{*}{ 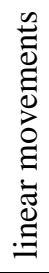 } & 1 & 0.008 & \multirow{19}{*}{0.074} & \multirow{19}{*}{0.018} \\
\hline & 2 & 0.007 & & \\
\hline & 3 & 0.010 & & \\
\hline & 4 & 0.015 & & \\
\hline \multirow{15}{*}{ 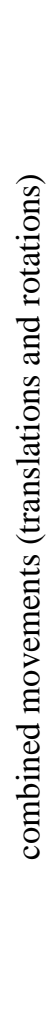 } & 5 & 0.022 & & \\
\hline & 6 & 0.018 & & \\
\hline & 7 & 0.018 & & \\
\hline & 8 & 0.019 & & \\
\hline & 9 & 0.019 & & \\
\hline & 10 & 0.018 & & \\
\hline & 11 & - & & \\
\hline & 12 & 0.023 & & \\
\hline & 13 & 0.003 & & \\
\hline & 14 & 0.008 & & \\
\hline & 15 & 0.012 & & \\
\hline & 16 & 0.013 & & \\
\hline & 17 & 0.014 & & \\
\hline & 18 & 0.028 & & \\
\hline & 19 & 0.014 & & \\
\hline
\end{tabular}

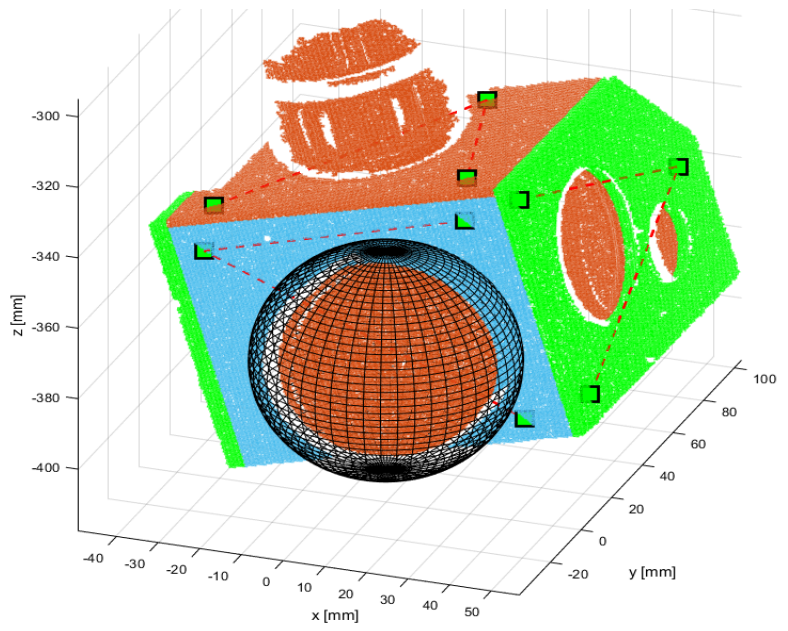

Fig. 15. Example of data selection to isolate points (blue) representing the front plane of the sample object. Each set of three squares selected on the point cloud was used to define the cut-off surface

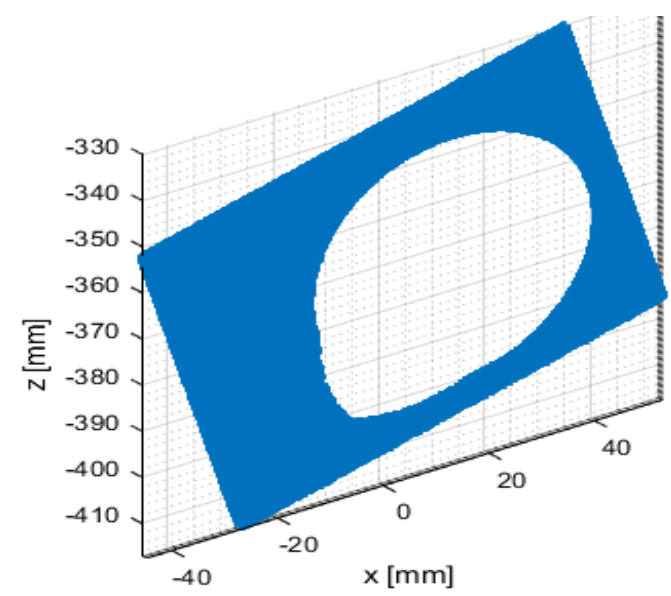

Fig. 16. Example of isolated points representing the front plane of the sample object for one of the scans

The flatness error for the combined scans is approximately $0.07 \mathrm{~mm}$, which again may be the result of inaccurate positioning of the $Y$ axis of the machine tool. It should be noted that the analysed plane was oriented perpendicular to the direction of the $Y$ axis. Although the parallelism index is satisfactory for each of the planes separately, their relative distance apart results in a large misalignment in the $Y$-axis direction. 


\section{CONCLUSION AND FUTURE WORKS}

The study showed that the concept of combining scans without using reference points and based on the movements of CNC machine axes is valid. The obtained errors are a combination of errors caused by the optical components together with the calibration technique on the machine tool.

Studies carried out proved the validity of our idea and the possibility of applying such a system in future machine tools provided a very good knowledge of the kinematic model reconstructed in the scanner workspace. It is worth mentioning, that the tests were a demonstration of the method of merging scans, for which the mounting location of the sample object did not matter. Although in this study the sample object was mounted in a spindle in order to obtain more complex transformations, an identical concept may be concerned in the case of the machining table, which provides a more stable positioning of the scanned components.

This article is an introduction to further research work aimed at scanning a workpiece without markers using a scanner mounted in the spindle of a machine tool. The scanner coordinate system and the $L C M S$ will take place at very two ends of the machine kinematic structure and thus the back-transformation of the scans to LMCS will be more complex. It will be also necessary to extend the calibration procedures for each axis using very accurately defined reference point $m$ located in a known position to the machine coordinate system $M$. In the future research we plan to test the scanner using the rotation of the spindle, which will increase the possibilities and range of observation of the workspace of the CNC machine by the scanner.

The presented method of fast geometry measurements in the same mounting as the machining process will provide new tools for the operator, enabling e.g., basing, planning machining allowance, or inter-process inspection. Although touch-trigger probe can achieve the accuracy of 2-3 $\mu \mathrm{m}$, we believe that the scanner as an interchangeable tool can replace it in the future, enabling autonomous and collision-free scanning of the workspace, including identification of fixtures, obstacles, etc. The technique could also eliminate the need to remove and transport the workpiece for external inspection, minimizing machine downtime.

Unfortunately, the use of a scanner does come with certain limitations that must be taken into account when developing a scanning system. The problem of scanning shiny surfaces is well known and scanning workpieces on the CNC machine tool can cause this problem. Covering the surface with matting spray may be difficult after or during machining due to the workpiece remaining wet as a result of cutting fluid application. In this case, future applications of artificial intelligence algorithms can be helpful in positioning the scanning head so that as much of the surface as possible can be scanned correctly by avoiding or minimising reflection effects.

\section{ACKNOWLEDGEMENTS}

The authors thank Krzysztof Filipowicz, an employee of the Faculty of Mechanical Engineering and Mechatronics of the West Pomeranian University of Technology in Szczecin, for his help in preparing and conducting tests on a CNC machine tool. 


\section{REFERENCES}

[1] RIYADI T., YULIANTO Y.H., EFFENDY M., SARJITO ZHEN Z., PING T.L., 2018, Evaluation on a Digitized CAD Model of 3D Scanner Used in Reverse Engineering, Conference: 12th South East Asian Technical University Consortium (SEATUC), DOI: 10.1109/SEATUC.2018.8788885.

[2] XU J., XI N., ZHANG C., SHI Q., GREGORY J., 2011, Real-Time 3D Shape Inspection System of Automotive Parts Based on Structured Light Pattern, Opt. Laser Technol., 43/1, 1-8, DOI: 10.1016/j.optlastec.2010.04.008.

[3] PATHAK V.K., SINGH A.K., SIVADASAN M., SINGH N.K., 2018, Framework for Automated GD\&T Inspection Using 3D Scanner, J. Inst. Eng. Ser. C, 99/2, 197-205, DOI: 10.1007/s40032-016-0337-7.

[4] HUANG W., KOVACEVIC R., 2011, A Laser-Based Vision System for Weld Quality Inspection, Sensors, 11/1, 506-521, DOI: 10.3390/s110100506.

[5] PIEDRA-CASCÓN W., MEYER M.J., METHANI M.M., REVILLA-LEÓN M., 2020, Accuracy (Trueness and Precision) of a Dual-Structured Light Facial Scanner and Interexaminer Reliability, The Journal of Prosthetic Dentistry, 124/5, 567-574, DOI: 10.1016/j.prosdent. 2019.10.010.

[6] LIN S.C.H., DOUGLASS M.J., HOLDAWAY S.J., FLOYD B., 2010, The Application of 3D Laser Scanning Technology to the Assessment of Ordinal and Mechanical Cortex Quantification in Lithic Analysis, J. Archaeol. Sci., 37/4, 694-702, DOI: 10.1016/j.jas.2009.10.030.

[7] ABOUHASHEM Y., DAYAL M., SAVANAH S., ŠTRKALJ G., 2015, The Application of 3D Printing in Anatomy Education, Medical Education Online, 20/1, Co-Action Publishing, DOI: 10.3402/meo.v20.29847.

[8] APEAGYEI P.R., 2010, Application of 3D Body Scanning Technology to Human Measurement for Clothing Fit, Int. J. Digit. Content Technol., 4/7, DOI: 10.4156/jdcta.vol4.issue7.6.

[9] KRAWIEC P., DOMEK G., WARGUŁA L., WALUŚ K., ADAMIEC J., 2018, The Application of the Optical System ATOS II for Rapid Prototyping Methods of Non-Classical Models of Cogbelt Pulleys, MATEC Web of Conferences, 157, 01010, DOI: 10.1051/matecconf/201815701010.

[10] WAGNER M., HESS P., REITELSHÖFER S., FRANKE J., 2016, 3D Scanning of Workpieces with Cooperative Industrial Robot Arms, 47th International Symposium on Robotics, ISR, 431-438.

[11] ZHANG X., LI W., LIOU F., 2018, Damage Detection and Reconstruction Algorithm in Repairing Compressor Blade by Direct Metal Deposition, Int. J. Adv. Manuf. Technol., 95/5-8, 2393-2404, DOI: 10.1007/s00170-0171413-8.

[12] MARTÍNEZ-PELLITERO S., CUESTA E., GIGANTO S., BARREIRO J., 2018, New Procedure for Qualification of Structured Light 3D Scanners Using an Optical Feature-Based Gauge, Opt. Lasers Eng., 110, 193-206, DOI: 10.1016/j.optlaseng.2018.06.002.

[13] EIRÍKSSON E.R., WILM J., PEDERSEN D.B., AANAES H., 2016, Precision and Accuracy Parameters in Structured Light 3-D Scanning, ISPRS, Int. Arch. Photogramm. Remote Sens. Spat. Inf. Sci., XL-5/W8/, 7-15, DOI: 10.5194/isprs-archives-xl-5-w8-7-2016.

[14] ADAMCZYK M., KAMIŃSKI M., SITNIK R., BOGDAN A., KARASZEWSKI M., 2014, Effect of Temperature on Calibration Quality of Structured-Light Three-Dimensional Scanners, Appl. Opt., 53/23, 51-54, DOI: 10.1364/ ao.53.005154.

[15] ADAMCZYK M., LIBERADZKI P., SITNIK R., 2020, Temperature Compensation Method for Mechanical Base of 3D-Structured Light Scanners, Sensors (Switzerland), 20/2, 362, DOI: 10.3390/s20020362.

[16] THE ASSOCIATION OF GERMAN ENGINEERS, 2012, VDI-Standard: VDI/VDE 2634 Optical 3-D Measuring Systems - Optical Systems Based on Area Scanning.

[17] JASIŃSKI D., GĘBARSKI K., 2013, Metrological Accuracy of the Non-Contact 3D Scanner According to the Standard VDI/VDE 2634 - Examples of Measurements with a Certified Polish 3D Scanner by SMARTTECH, XII Forum Inżynierskie ProCAx, 87, 8, (in Polish).

[18] JECIĆ S., DRVAR N., 2003, The Assessment of Structured Light and Laser Scanning Methods in 3D Shape Measurements, Proc. 4th Int. Congr. Croat. Soc. Mech., 237-244.

[19] GUERRA M.G., LAVECCHIA F., MAGGIPINTO G., GALANTUCCI L.M., LONGO G.A., 2019, Measuring Techniques Suitable for Verification and Repairing of Industrial Components: A Comparison Among Optical Systems, CIRP J. Manuf. Sci. Technol., 27, 114-123, DOI: 10.1016/j.cirpj.2019.09.003.

[20] ISA M.A., LAZOGLU I., 2017, Design and Analysis of a 3D Laser Scanner, Meas. J. Int. Meas. Confed., 111, 122133, DOI: 10.1016/j.measurement.2017.07.028.

[21] CHROMY A., 2015, High-Accuracy Volumetric Measurements of Soft Tissues Using Robotic 3D Scanner, IFACPapersOnLine, 28/4, 318-323, DOI: 10.1016/j.ifacol.2015.07.054.

[22] GESSNER A., STANIEK R., BARTKOWIAK T., 2015, Computer-Aided Alignment of Castings and Machining Optimization, Proc. Inst. Mech. Eng. Part C, J. Mech. Eng. Sci., 229/3, 485-492, DOI: 10.1177/0954406214536380. 
[23] PAJOR M., GRUDZIŃSKI M., 2015, Intelligent Machine Tool - Vision Based 3D Scanning System for Positioning of the Workpiece, Solid State Phenom., 220-221, 497-503, DOI: 10.4028/www.scientific.net/SSP.220-221.497.

[24] OKARMA K., GRUDZIŃSKI M., 2012, The 3D Scanning System for the Machine Vision Based Positioning of Workpieces on the CNC Machine Tools, 17th International Conference on Methods and Models in Automation and Robotics, MMAR, 85-90, DOI: 10.1109/MMAR.2012.6347906.

[25] YU Z., WANG T., WANG P., TIAN Y., LI H., 2019, Rapid and Precise Reverse Engineering of Complex Geometry through Multi-Sensor Data Fusion, IEEE Access, 7, 165793-165813, DOI: 10.1109/ACCESS.2019.2948124.

[26] SONG L., SUN S., YANG Y., ZHU X., GUO Q., YANG H., 2019, A Multi-View Stereo Measurement System Based on a Laser Scanner for Fine Workpieces, Sensors, Switzerland, 19/2, 381, DOI: 10.3390/s19020381.

[27] https://www.cncstepusa.com/cnc-laser-scanner-4500.

[28] CHANG W.Y., HSU J.W., HSU B. Y., 2019, 3D Scanning System of Structured Light for Aiding Workpiece Position of CNC Machine Tool, Proceedings of the 2018 IEEE International Conference on Advanced Manufacturing, ICAM, 388-391, DOI: 10.1109/AMCON.2018.8614757.

[29] SRINIVASAN H., HARRYSSON O.L.A., WYSK R.A., 2015, Automatic Part Localization in a CNC Machine Coordinate System by Means of 3D scans, Int. J. Adv. Manuf. Technol., 81/5-8, 1127-1138, DOI: 10.1007/s00170015-7178-z.

[30] ZHAO H., et al., 2013, The in-Situ 3D Measurement System Combined with CNC Machine Tools, International Conference on Optics in Precision Engineering and Nanotechnology icOPEN2013, 8769, 876912, DOI: 10.1117/ 12.2021111.

[31] FRÖHLICH M., 2012, Method and Device for Determining an Effective Vent, EP 2759822 B1.

[32] JENS O., MANUEL K., 2015, Method, Machining Unit And Computer Program Product for the Image-Based Positioning of Workpiece Machining Operations, EP 3108311 B1.

[33] DOLD P., et al., 2014, Validation of an Optical System to Measure Acetabular Shell Deformation in Cadavers, Proc. Inst. Mech. Eng. Part H, J. Eng. Med., 228/8, 781-786, DOI: 10.1177/0954411914546562.

[34] OOMORI S., NISHIDA T., KUROGI S., 2016, Point Cloud Matching Using Singular Value Decomposition, Artif. Life Robot., 21/2, 149-154, DOI: 10.1007/s10015-016-0265-x.

[35] THE AMERICAN SOCIETY OF MECHANICAL ENGINEERS, 2018, ASME-Standard: Y14.5 Dimensioning and Tolerancing. 